\title{
Preface
}

\section{Anesthesiologists in Times of \\ Disaster: A Rich History, a Busy}

Future

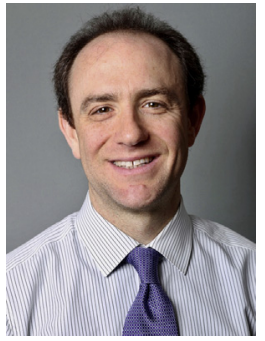

Jesse Raiten, MD

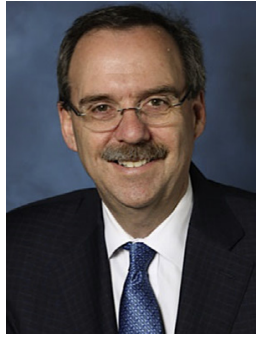

Lee A. Fleisher, MD, FACC, FAHA

Editors

When the COVID-19 pandemic struck the United States in the early months of 2020, our national health care system was thrust into one of the most challenging periods it has faced in recent history. Over the ensuing months, millions of people fell ill, and hundreds of thousands died. Hospital facilities, particularly critical care resources, were stretched to capacity and beyond. It has been said that necessity is the mother of invention, and novel strategies and staffing paradigms were developed to not only provide medical care to patients but also maximize staff utilization and provide additional support where needed.

The health care community's response to COVID-19 was a tremendous team effort, bringing together professionals from every specialty and service. It comes as no surprise that anesthesiologists and anesthesia providers assumed central roles in the response, not only in the United States but also on a global scale. Anesthesiologists assumed care for critically ill patients on ventilators, developed novel strategies to safely intubate patients, led procedure teams, assisted overworked respiratory therapists, and provided critical council to hospital administrators as clinical landscapes radically shifted to accommodate a new patient population.

The role of anesthesiologists in times of disaster has a rich history, from the battlefields of World War II to the field hospitals and make-shift operating rooms that have accompanied virtually every natural disaster in recent times. Anesthesiologists, by virtue of their broad training in the care of critically ill patients, wide range of procedural skills, and proficiency with patient triage and risk assessment, are well suited to lead institutional and regional medical efforts in times of crisis.

In this issue of Anesthesiology Clinics, authors explore the vast roles that anesthesiologists play in times of disaster. We consider the fundamental requirements for a 
successful disaster response plan, be it to respond to a natural or manmade disaster. A diverse group of authors from across the globe, including both developed and resource-limited countries, describes how they handled the COVID-19 pandemic, from China to Italy to Nepal. We then take an in-depth look at how anesthesiologists can lead in times of physical disasters, as well as in the unique environments of mass casualty events, war zones, and military special operations. Recognizing that health care systems have many mandates apart from direct patient care, we explore how resident education, as well as research activities, may be influenced and modified in times of calamity.

There are many threats facing the world today. Climate change has led to increasing weather-related disasters. Population expansion and human encroachment on animals' natural habitats could potentially lead to further pandemic events. Expansion of military capabilities, including weapons of mass destruction, has the potential to cause large-scale casualties both in urban areas and on the battlefield. In all of these cases, our health care systems will require strong leadership and creative, adaptive physicians and staff to navigate unchartered waters. There is no physician better positioned to lead through these challenging times than an anesthesiologist.

Jesse Raiten, MD Department of Anesthesiology and Critical Care Perelman School of Medicine of the University of Pennsylvania 3400 Spruce Street, Dulles 6 Philadelphia, PA 19104, USA

Lee A. Fleisher, MD, FACC, FAHA Perelman School of Medicine University of Pennsylvania 3400 Spruce Street, Dulles 680 Philadelphia, PA 19104, USA

E-mail addresses: 